\title{
Efficacy of Interdental Brush as an Adjunct to Manual Toothbrush on Gingival Status in Patients undergoing Fixed Orthodontic Therapy: A Clinical Study
}

\author{
${ }^{1}$ Rao Anupama, ${ }^{2}$ Shah Seema, ${ }^{3}$ Kashyap R Shankar, ${ }^{4}$ Rajesh Hosadurga, ${ }^{5}$ Kumar Vijaya K, ${ }^{6}$ Boloor Vinita A
}

\begin{abstract}
Aim: Aim of the study was to evaluate the efficacy of manual toothbrush (STIM ${ }^{\circledR} 42$ Orthodontic toothbrush) with or without an interdental brush (IDB) (STIM ${ }^{\circledR}$ PROXA-Extra fine) on gingival status in patients undergoing fixed orthodontic therapy.

Materials and methods: In a randomized control trial of 60 subjects undergoing orthodontic therapy with Begg's appliance enrolled for the study. They were placed in a maintenance regimen with (STIM ${ }^{\circledR} 42$ orthodontic toothbrush) after thorough scaling and root planning (SRP) for four weeks before the commencement of the study. This was done to attain uniformity and avoid bias in both the groups at the baseline. At the end of four weeks, subjects were randomly divided into two groups, group I-30 subjects used STIM ${ }^{\circledR} 42$ Orthodontic toothbrush only and group II-30 subjects used STIM $^{\circledR}$ PROXA-extra fine as an adjunct to STIM ${ }^{\circledR} 42$ Orthodontic toothbrush for four weeks. Clinical parameters such as bonded bracket plaque index (BBPI), gingival index (GI), and gingival bleeding index (GBI) were recorded at baseline, 1, 2 and 4 weeks. Analysis of data was done using repeated measures analysis of variance (ANOVA) and unpaired student t-test using Statistical Package for the Social Sciences (SPSS) 22 software.
\end{abstract}

Results: Result of the study showed a statistically significant reduction $(p<0.001)$ in BBPI, GI and GBI in group. II subjects when compared to group. I.

Conclusion: Manual orthodontic brush along with IDB proved to be superior, in controlling plaque, gingival inflammation and gingival bleeding in subjects undergoing fixed orthodontic treatment.

Clinical significance: Our study supports the use of interdental brushes along with manual toothbrush during fixed orthodontic treatment. It can significantly improve plaque control, thereby improving gingival health. To promote gingival health dentists need to motivate and reinforce this beneficial practice among their patients undergoing fixed orthodontic treatment.

Keywords: Fixed orthodontic therapy, Interdental brush, Manual toothbrush, Plaque.

1-3,5,6 Department of Periodontology, Yenepoya Dental College, Yenepoya, Mangaluru, Karnataka, India

${ }^{4}$ Department of Periodontics, Faculty of Dentistry, Melaka Manipal Medical College, Bukit Baru, Melaka, Malaysia

Corresponding Author: Rajesh Hosadurga, Department of Periodontics, Faculty of Dentistry, Melaka Manipal Medical College, Bukit Baru, Melaka, Malaysia, Phone: (606) 2896662, e-mail: rajesh.hosadurga@manipal.edu.my
How to cite this article: Anupama R, Seema S, Shankar KR, Hosadurga R, Vijaya KK, Vinita BA. Efficacy of Interdental Brush as an Adjunct to Manual Toothbrush on Gingival Status in Patients undergoing Fixed Orthodontic Therapy: A Clinical Study. World J Dent 2018;9(5):355-360.

\section{Source of support: Nil}

Conflict of interest: None

\section{INTRODUCTION}

Dental plaque as the biofilm is a prime etiological factor responsible for gingival inflammation and progression of periodontal disease. ${ }^{1}$ Studies have shown that fixed orthodontic brackets influence the accumulation of plaque and the colonization of bacteria resulting in increased inflammation and bleeding. ${ }^{2}$ Level of oral hygiene during the orthodontic treatment has a direct impact on periodontal health. Subjects with fixed orthodontic therapy, often show inadequate plaque control due to inability to perform optimum oral hygiene practices with orthodontic appliances in place. Tooth brushing is the most commonly used tool for plaque removal, other aids such as powered toothbrush, dental floss, IDB, toothpicks and oral irrigation devices are also used, but much less commonly. ${ }^{3}$ Although regular toothbrush is successful in removing plaque from specific tooth surfaces, it cannot completely remove plaque around and beneath the brackets. As an adjunct to regular brushing, IDB, which are small-headed brushes, provide better access behind the archwire and reduce plaque accumulation than tooth brushing alone. ${ }^{4}$ They seem to be much simpler and quicker compared to flossing and other interdental aids. ${ }^{5}$ Till date, randomized clinical trials to strengthen the evidence and support the recommendation of IDB for orthodontic patients is sparse. ${ }^{6}$ Hence, the objective of the study was to do a comparative evaluation of the efficacy of manual tooth brushing with or without IDB on gingival status in patients undergoing fixed orthodontic therapy.

\section{MATERIALS AND METHODS}

A single centered, investigator blind, parallel group, randomized control clinical study of four weeks duration was done in accordance with the Consolidated Standards of Reporting Trials (CONSORT) guidelines. The study was 
undertaken after the clinical protocol and informed consent form was reviewed and approved by the Institutional Ethical committee (YUEC/2013/063). Written informed consent was taken from all the subjects at the beginning of the study. All the participants were selected from the outpatients reporting to the department of orthodontics.

The estimated sample size from previous studies was 60 subjects. However, considering a 10\% drop out rate, we selected 70 subjects randomly using computer-generated random numbers, from the outpatient, department of orthodontics. Only 64 participants agreed to enroll for the study. All the subjects undergoing active fixed orthodontic treatment involving full upper and lower arch with Begg's appliance, with a minimum of 20 scorable permanent teeth were randomly selected using computergenerated random numbers, and allocated to two groups. The exclusion criteria were: (a) subjects with periodontitis (b) compromised manual dexterity (c) history of antibiotics and anti-inflammatory drugs in last six months (d) habits like tobacco smoking.

Once enrolled, all the subjects used a manual orthodontic toothbrush (STIM ${ }^{\circledR} 42$ orthodontic toothbrush, Global Dent Aids Pvt. Ltd, India) and Colgate ${ }^{\circledR}$ toothpaste (Colgate-Palmolive Pvt. Ltd, India). All the subjects were instructed to refrain from using any additional oral hygiene aids until the end of the study period. They were taught modified bass technique brushing by a dental assistant not involved in the study. They were asked to brush for two minutes (30 sec/quadrant), twice a day, with the orthodontic brush and the dentifrice provided to them. Scaling and polishing were performed for all the subjects. All of them received verbal and written instructions on oral hygiene maintenance in their respective language. They were placed under supervision for four weeks before the commencement of the study. This was done to attain uniformity and avoid bias in both the groups at the baseline. At the end of 4 weeks, four patients failed to turn up for a scheduled appointment. Hence, 60 subjects were assigned to two groups (30 in each group) after 4 weeks by handing over the computer-generated random number concealed in a sealed envelope by the dental assistant not associated with the study.

Subjects in group I was instructed by the dental assistant to continue the modified Bass tooth brushing technique for the next four weeks. Subjects in the group II were given additional IDB (STIM PROXA-Extra fine, Global Dent Aids Pvt Ltd, India) to be used twice a day as an adjunct to existing orthodontic brush. Use of IDB for group II subjects was demonstrated on a model.

The product used by the subjects was supervised by a dental assistant at the baseline visit, to ensure that the product was used properly. Subjects in group II were asked to bring their IDB on every visit and assessed for its use.

Hence, group I-30 subjects used STIM ${ }^{\circledR} 42$ Orthodontic toothbrush only and group II-30 subjects used STIM $^{\circledR}$ PROXA-Extra fine as an adjunct to STIM $^{\circledR} 42$ orthodontic toothbrush.

The clinical parameters such as $\mathrm{BBPI}^{7}$ by Kilicoglu, gingival index $(\mathrm{GI})^{8}$ by Loe and Sillness and $\mathrm{GBI}^{9}$ by Ainamo and Bay for both the groups were recorded on 0 day (baseline value) followed by, one (7 days), two (14 days), and four (28 days) weeks by the principal investigator (Fig. 1). During the study period subjects had to refrain from any elective, nonemergency dental care including oral prophylaxis.

\section{RESULTS}

Statistical analysis was performed using IBM Statistical Program for Social Sciences IBM ${ }^{\circledR}$ SPSS $^{\circledR}$ Statistics V22.0 (SPSS Inc., Chicago Illinois, USA). Intragroup comparisons were analyzed by repeated measures ANOVA. Mean, SD, CI, and F value were tabulated. The Bonferroni correction was done to adjust pro ability $(\mathrm{p})$ values to avoid the increased risk of type I error when making multiple comparisons. ${ }^{10}$ The intergroup comparisons were analyzed by unpaired student ' $t$ ' test. A $p<0.05$ was considered statistically significant.

A total of 60 participants $(n=60)$ with $n=30$ in each group were enrolled in the study. The mean BBPI, mean GI, mean GBI scores were analyzed.

Mean values of all the clinical parameters for group I and group II with standard deviation is shown in Table 1. There was no statistically significant difference in Groups I and II at baseline. Intragroup comparison of BBPI, GI, GBI differs significantly at one, two, four weeks for group I subjects using an orthodontic toothbrush (STIM ORTHO) and group II subjects using an orthodontic toothbrush (STIM ORTHO) and an IDB (STIM PROXA-Extra fine) $(\mathrm{p}<0.001)$.

\section{Intragroup Comparisons}

\section{Gingival Bleeding Index}

There was a statistically significant increase in mean scores in group I from baseline $(12.063 \pm 3.336)$ to four weeks $(28.626 \pm 8.697)$ (Table 1$)$.

\section{Gingival Index}

There was a statistically significant increase in the mean scores in both group I baseline $(0.306 \pm 0.132)$ to four weeks $(1.093 \pm 0.510)$ and group II baseline $(0.279 \pm 0.126)$ to four weeks $(0.327 \pm 0.119)$. Mean GI scores were higher in group I at the end of four weeks (Table 1). 


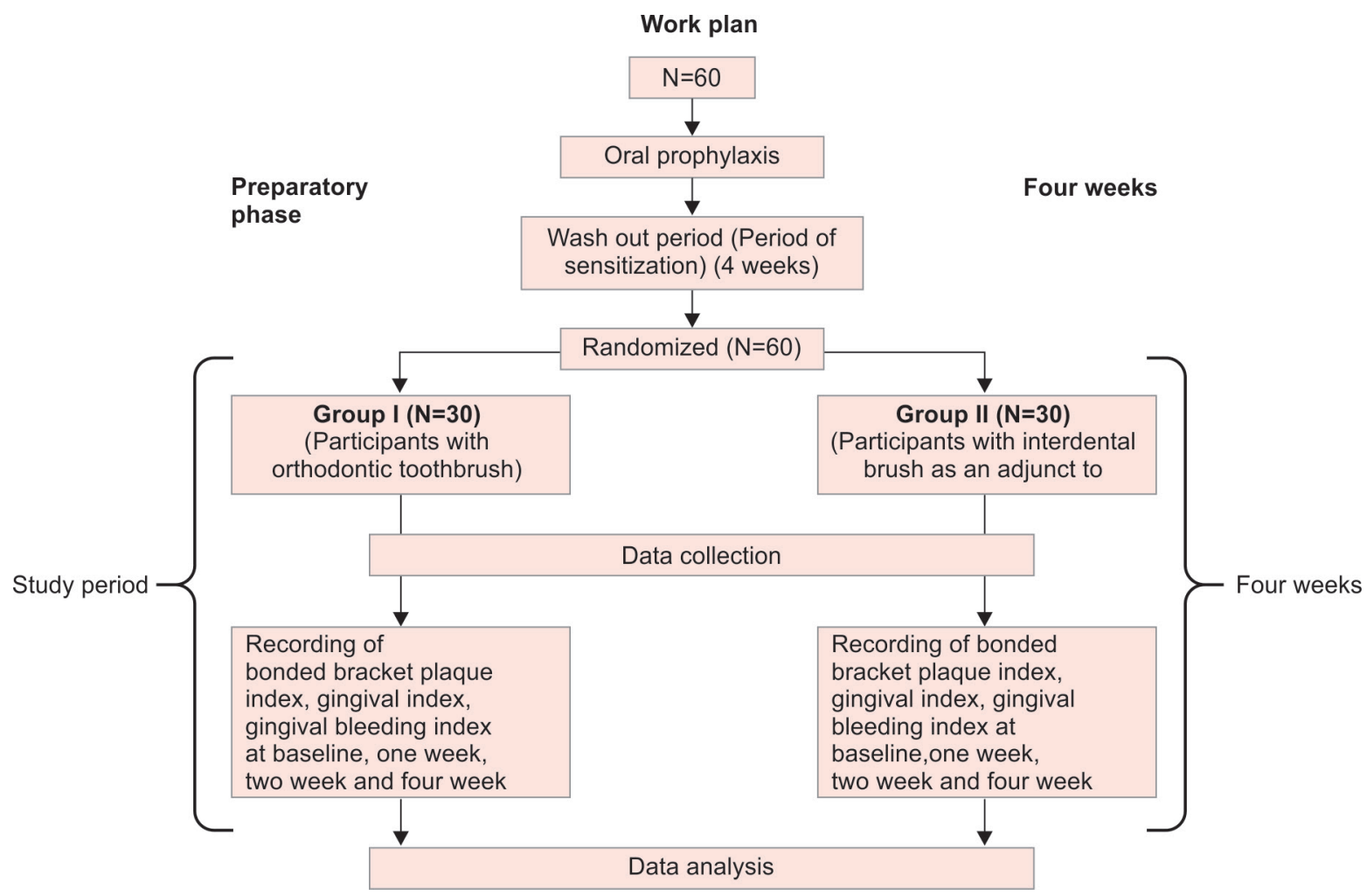

Fig. 1: Flow chart showing study design

Table 1: Intra group comparison of mean scores (SD) of GBI, GI and BBPI in group I and II at baseline, one week, two week and four weeks

\begin{tabular}{|c|c|c|c|c|c|}
\hline & & Mean $\pm S D$ & C.I & $\begin{array}{l}\text { Repeated measures } \\
\text { ANOVA F value }\end{array}$ & $p$-value \\
\hline \multicolumn{6}{|l|}{ BBPI } \\
\hline \multirow[t]{4}{*}{ Group I } & Baseline & $0.43 \pm 0.10$ & $(0.4-0.47)$ & 110.711 & $0.000^{*}$ \\
\hline & One week & $0.61 \pm 0.16$ & $(0.55-0.67)$ & & \\
\hline & Two weeks & $0.87 \pm 0.38$ & $(0.73-1.01)$ & & \\
\hline & Four weeks & $1.71 \pm 0.54$ & $(1.51-1.92)$ & & \\
\hline \multirow[t]{4}{*}{ Group II } & Baseline & $0.44 \pm 0.10$ & $(0.4-0.47)$ & 5.382 & $0.002^{*}$ \\
\hline & One week & $0.46 \pm 0.097$ & $(0.43-0.51)$ & & \\
\hline & Two weeks & $0.48 \pm 0.11$ & $(0.44-0.53)$ & & \\
\hline & Four weeks & $0.48 \pm 0.11$ & $(0.43-0.52)$ & & \\
\hline \multicolumn{6}{|l|}{ GI } \\
\hline \multirow[t]{4}{*}{ Group I } & Baseline & $0.31 \pm 0.13$ & $(0.26-0.36)$ & 59.828 & $0.000^{*}$ \\
\hline & One week & $0.48 \pm 0.29$ & $(0.37-0.59)$ & & \\
\hline & Two weeks & $0.67 \pm 0.42$ & $(0.51-0.82)$ & & \\
\hline & Four weeks & $1.09 \pm 0.51$ & $(0.9-1.28)$ & & \\
\hline \multirow[t]{4}{*}{ Group II } & Baseline & $0.28 \pm 0.13$ & $(0.23-0.33)$ & 14.870 & $0.000^{*}$ \\
\hline & One week & $0.31 \pm 0.12$ & $(0.26-0.35)$ & & \\
\hline & Two weeks & $0.31 \pm 0.11$ & $(0.27-0.35)$ & & \\
\hline & Four weeks & $0.32 \pm 0.11$ & $(0.28-0.37)$ & & \\
\hline \multicolumn{6}{|l|}{$G B I$} \\
\hline \multirow[t]{4}{*}{ Group I } & Baseline & $12.01 \pm 3.33$ & $(10.82-13.31)$ & 74.041 & $0.000^{*}$ \\
\hline & One week & $15.36 \pm 4.21$ & $(13.79-16.94)$ & & \\
\hline & Two weeks & $19.84 \pm 6.56$ & (17.39-22.29) & & \\
\hline & Four weeks & $28.63 \pm 8.70$ & $(25.38-31.87)$ & & \\
\hline \multirow[t]{4}{*}{ Group II } & Baseline & $11.39 \pm 2.33$ & $(10.53-12.27)$ & 1.553 & $0.207 \#$ \\
\hline & One week & $11.40 \pm 2.43$ & $(10.49-12.31)$ & & \\
\hline & Two weeks & $11.12 \pm 2.40$ & $(10.22-12.01)$ & & \\
\hline & Four weeks & $11.11 \pm 2.60$ & $(10.15-12.08)$ & & \\
\hline
\end{tabular}

*-Highly significant, \#-Non significant 


\section{Bonded Bracket Plaque Index (BBPI)}

There was a statistically significant increase in the mean scores in both group I baseline $(0.435 \pm 0.101)$ to four weeks $(1.714 \pm 0.541)$ and group II baseline $(0.435 \pm 0.101)$ to four weeks $(0.476 \pm 0.111)$. Mean BBPI were higher in group I at the end of four weeks (Table 1).

After Bonferroni correction, there was a statistically significant difference in the mean scores of all the clinical parameters (Table 2).

\section{Intergroup Comparisons}

There was no significant difference in mean scores of GBI, GI, and BBPI at the baseline between group I and group II (Table 3).
There was a significant difference in the mean scores of GBI, GI, and BBPI at one week, two week and four weeks between group I and group II (Table 3).

\section{DISCUSSION}

Plaque accumulation is an inevitable process in patients undergoing fixed orthodontic therapy in spite of advances in orthodontic appliances. Constant efforts are needed by the patients to mechanically disrupt the biofilm from the plaque retentive surfaces to maintain good oral hygiene throughout the orthodontic treatment. ${ }^{11}$ During orthodontic therapy, it's quite challenging task for the patients to remove complete plaque using a regular toothbrush due to either lack of knowledge about an availability of oral hygiene aids or negligence by patient's themselves. ${ }^{12}$

Table 2: Bonferroni comparison for Intra group comparison of mean scores (SD) of GBI, GI and BBPI in group

\begin{tabular}{|c|c|c|c|c|c|}
\hline & & Mean difference & $S D$ of difference & Change (\%) & $\begin{array}{l}\text { Bonferroni test } \\
\text { p-value }\end{array}$ \\
\hline \multicolumn{6}{|l|}{$\overline{B B P I}$} \\
\hline \multirow[t]{6}{*}{ Group I } & Baseline-one week & -0.171 & 0.144 & -39.36 & $0.000^{*}$ \\
\hline & Baseline-two week & -0.434 & 0.348 & -99.62 & $0.000^{*}$ \\
\hline & Baseline-four week & -1.279 & 0.549 & -293.80 & $0.000^{*}$ \\
\hline & One to two weeks & -0.262 & 0.296 & -43.24 & $0.000^{*}$ \\
\hline & One to four weeks & -1.108 & 0.495 & -182.58 & $0.000^{*}$ \\
\hline & Second to four week & -0.845 & 0.521 & -97.28 & $0.000^{*}$ \\
\hline \multirow[t]{6}{*}{ Group II } & Baseline-one week & -0.034 & 0.072 & -7.81 & $0.015^{*}$ \\
\hline & Baseline-two week & -0.049 & 0.098 & -11.26 & $0.010^{*}$ \\
\hline & Baseline-four week & -0.040 & 0.098 & -9.26 & $0.031^{*}$ \\
\hline & One to two weeks & -0.015 & 0.050 & -3.20 & $0.687 \#$ \\
\hline & One to four weeks & -0.006 & 0.050 & -1.35 & $1.000 \#$ \\
\hline & Second to four week & 0.009 & 0.038 & 1.79 & $1.000 \#$ \\
\hline \multicolumn{6}{|c|}{ 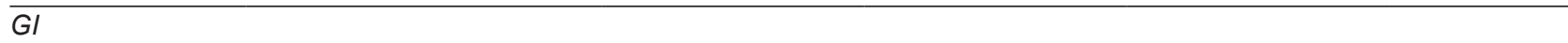 } \\
\hline \multirow[t]{6}{*}{ Group I } & Baseline-one week & -0.172 & 0.255 & -56.21 & $0.005^{\star}$ \\
\hline & Baseline-two week & -0.361 & 0.408 & -117.86 & $0.000^{*}$ \\
\hline & Baseline-four week & -0.787 & 0.463 & -257.08 & $0.000^{*}$ \\
\hline & One to two weeks & -0.189 & 0.217 & -39.47 & $0.000^{*}$ \\
\hline & One to four weeks & -0.615 & 0.321 & -128.59 & $0.000^{*}$ \\
\hline & Second to four week & -0.426 & 0.304 & -63.90 & $0.000^{*}$ \\
\hline \multirow[t]{6}{*}{ Group II } & Baseline-one week & -0.030 & 0.037 & -10.77 & $0.001^{*}$ \\
\hline & Baseline-two week & -0.032 & 0.040 & -11.36 & $0.001^{*}$ \\
\hline & Baseline-four week & -0.049 & 0.048 & -17.46 & $0.000^{*}$ \\
\hline & One to two weeks & -0.002 & 0.025 & -0.54 & $1.000 \#$ \\
\hline & One to four weeks & -0.019 & 0.048 & -6.05 & $0.252 \#$ \\
\hline & Second to four week & -.017 & 0.042 & -5.48 & $0.197 \#$ \\
\hline \multicolumn{6}{|l|}{$\overline{G B I}$} \\
\hline \multirow[t]{6}{*}{ Group I } & Baseline-one week & -3.300 & 2.812 & -27.36 & $0.000^{*}$ \\
\hline & Baseline-two week & -7.779 & 5.552 & -64.48 & $0.000^{*}$ \\
\hline & Baseline-four week & -16.563 & 8.143 & -137.30 & $0.000^{*}$ \\
\hline & One to two weeks & -4.479 & 4.230 & -29.15 & $0.000^{*}$ \\
\hline & One to four weeks & -13.263 & 8.295 & -86.33 & $0.000^{*}$ \\
\hline & Second to four weeks & -8.784 & 7.681 & -44.27 & $0.000^{*}$ \\
\hline \multirow[t]{6}{*}{ Group II } & Baseline-one week & -0.004 & 1.055 & -0.04 & $1.000 \#$ \\
\hline & Baseline-two week & 0.283 & 1.094 & 2.48 & $1.000 \#$ \\
\hline & Baseline-four week & 0.285 & 1.405 & 2.50 & $1.000 \#$ \\
\hline & One to two weeks & 0.287 & 0.712 & 2.51 & $0.213 \#$ \\
\hline & One to four weeks & 0.289 & 1.054 & 2.54 & $0.862 \#$ \\
\hline & Second to four weeks & 0.003 & 0.647 & 0.02 & $1.000 \#$ \\
\hline
\end{tabular}

*-significant, \#-non significant 
Table 3: Inter group comparison of mean scores (SD) of GBI, GI and BBPI in group I and II at baseline, baseline, one week, two week and four week

\begin{tabular}{|c|c|c|c|c|}
\hline & $\begin{array}{l}\text { Group I } \\
(\text { Mean } \pm \text { SD) }\end{array}$ & $\begin{array}{l}\text { Group II } \\
\text { (Mean } \pm \text { SD) }\end{array}$ & $t$ test & $p$ \\
\hline \multicolumn{5}{|l|}{$B B P I$} \\
\hline Baseline & $0.43 \pm 0.10$ & $0.43 \pm 0.10$ & 0.000 & $1.00 \#$ \\
\hline One week & $0.60 \pm 0.16$ & $0.46 \pm 0.97$ & 3.99 & $0.000^{*}$ \\
\hline Two weeks & $0.87 \pm 0.37$ & $0.48 \pm 0.11$ & 5.34 & $0.000^{*}$ \\
\hline Four weeks & $1.71 \pm 0.54$ & $0.47 \pm 0.11$ & 12.28 & $0.000^{*}$ \\
\hline \multicolumn{5}{|l|}{ GI } \\
\hline Baseline & $0.30 \pm 0.13$ & $0.27 \pm 0.12$ & 0.822 & $0.415 \#$ \\
\hline One weeks & $0.47 \pm 0.29$ & $0.30 \pm 0.11$ & 2.88 & $0.000^{*}$ \\
\hline Two weeks & $0.67 \pm 0.41$ & $0.31 \pm 0.12$ & 4.47 & $0.000^{*}$ \\
\hline Four weeks & $1.09 \pm 0.51$ & $0.32 \pm 0.11$ & 8.01 & $0.000^{*}$ \\
\hline \multicolumn{5}{|l|}{$G B I$} \\
\hline Baseline & $12.06 \pm 3.33$ & $11.39 \pm 2.33$ & 0.894 & $0.375 \#$ \\
\hline One weeks & $15.36 \pm 4.21$ & $11.40 \pm 2.43$ & 4.453 & $0.000^{*}$ \\
\hline Two weeks & $19.84 \pm 6.55$ & $11.11 \pm 2.39$ & 6.843 & $0.000^{*}$ \\
\hline Four weeks & $28.62 \pm 8.70$ & $11.11 \pm 2.59$ & 10.570 & $0.000^{*}$ \\
\hline
\end{tabular}

*-highly significant, \#-nonsignificant

In our study, we selected BBPI for plaque assessment as it is more relevant in presence orthodontic appliance where traditional plaque indices were unable to quantify the plaque distribution on an orthodontic bracket and braces. ${ }^{13}$

In comparison, both the groups did not show any significant difference in mean scores of GBI, GI, and BBPI at baseline. This could be attributed to the fact that in the present study both the groups received oral hygiene instructions and were placed in maintenance regimen for four weeks before the commencement of the study and periodic oral hygiene assessment. This helped to attain uniformity and avoid bias in both the groups.

On intragroup comparison, there was a significant increase in the mean scores of GI and BBPI from baseline to 28 days in groups I and II. These observations are similar to a study by Kenan et al. ${ }^{14}$ which showed orthodontic treatment with a fixed appliance increased the risk of poor oral hygiene status. A study by Ristic et al. ${ }^{15}$ of three-month duration also observed an increase in clinical and microbiological parameters in patients with fixed orthodontic appliances.

The GBI in group II showed no statistically significant difference from baseline to 28 days. Intergroup comparison showed that PI, GI, GBI were higher in group I subjects at one week, two weeks and four weeks as compared to group II subjects. This could be because of the use of interdental brushes facilitated better plaque removal. It was noted that the insertion of the IDB (STIM PROXAExtra fine) underneath the archwire seemed to be easier than the insertion of the manual toothbrush $\left(\right.$ STIM $^{\circledR} 42$ Orthodontic toothbrush) by group II subjects. Hence, less gingival inflammation and gingival bleeding were seen in group II subjects. These results are in accordance with the study by Wolff $\mathrm{D}^{16}$ who observed the use of both conventional and triangular IDB design reduced plaque scores equally in patients with multibracket appliances.

A systematic review by Goh $\mathrm{HH}^{5}$ stated that present practice of recommending the use of interdental/interspace brushes in addition to standard toothbrushes is not supported by clinical investigations and hence more studies are required to validate its use. This justifies the current study. The authors also reported that the extent of toothbrush wear increased during orthodontic treatment thereby the need to replace them more frequently. This would increase the economic burden of oral hygiene products for the patient. In the present study, a significant reduction in gingival inflammation was observed when interdental brushes were used as an adjunct to manual orthodontic brush thereby confirming the clinical benefit. However, a cost-benefit ratio was not carried out. This issue may be addressed in wellcontrolled longitudinal studies in the future.

Poklepovic et al. ${ }^{17}$ in a systematic review (2013) included the study by Jared et al. ${ }^{18}$ where he analyzed whether tooth brushing with interdental brushing was better than tooth brushing alone, and found very low-quality evidence for a reduction in gingivitis and plaque at one month. There was also a lack of sufficient evidence to determine whether interdental brushing was better than flossing. It may also be noted that in the current study, repeated oral hygiene instructions were given and the products used were supervised periodically to ensure correct usage. Hence, this might have resulted in the better clinical outcome.

Though motivation and supervision may be a crucial factor for patients undergoing fixed orthodontic therapy, use of interdental brush along with manual orthodontic brush improved the clinical parameters in the present study. 
Limitation of the study includes short-term (4 weeks) evaluation, the influence of 'Hawthorne effect' which may be due to the introduction of interdental brush for the first time for group II participants. Multicentered longitudinal studies are needed to increase the quality of evidence to confirm the efficacy of interdental brush in the maintenance of gingival health during fixed orthodontic therapy.

\section{CONCLUSION}

Patients with an inadequate oral hygiene regime during fixed orthodontic therapy are susceptible to periodontal disease, halitosis, and caries. Maintaining good oral hygiene is a challenging task among patients undergoing fixed appliance orthodontic treatment. Manual orthodontic brush along with IDB proved to be superior, in controlling plaque, gingival inflammation and gingival bleeding in patients undergoing fixed orthodontic treatment.

\section{CLINICAL SIGNIFICANCE}

Our study supports the use of interdental brushes along with manual toothbrush during fixed orthodontic treatment. It can significantly improve plaque control, thereby improving gingival health. Dentists need to motivate and reinforce this beneficial practice among their patients undergoing fixed orthodontic treatment.

\section{REFERENCES}

1. Cardoso Mde A, Saraiva P P, Maltagliati L Á, Rhoden FK, Costa CC, Normando D. et al. Alterations in plaque accumulation and gingival inflammation promoted by treatment with self-ligating and conventional orthodontic brackets. Dental Press J Orthod 2015;20:35-41.

2. Naranjo AA, Triviño ML, Jaramillo A, Betancourth M, Botero JE. Changes in the subgingivalmicrobiota and periodontal parameters before and 3 months after bracket placement. Am J Orthod Dentofacial Orthop. 2006;130(3):275.e17-22.

3. Singh T, Bhatia B, Singh G, Prasad PN. Comparative study of the plaque removal efficacy of three types of toothbrushes in patients with fixed orthodontic appliances J. Ind Orthod Soc. 2011;45(2):61-67.
4. Bock NC, von Bremen J, Kraft M, Ruf S. Plaque control effectiveness and handling of interdental brushes during multibracket treatment-a randomized clinical trial. European J Orthod. 2010:32(4):408-413.

5. Imai PH, Hatzimanolakis PC. Encouraging client compliance for interdental care with the interdental brush: The client's perspective. Can J Dent Hyg .2010;44(2):71-75.

6. Goh HH. Interspace/interdental brushes for oral hygiene in orthodontic patients with fixed appliances. Cochrane Database Syst Rev. 2007;18(3):CD005410.

7. Al-Anezi SA, Harradine NW. Quantifying plaque during orthodontic treatment: A systematic review. Angle Orthod. 2012;82(4):748-753.

8. Löe H, Silness J. Periodontal disease in pregnancy. I. Prevalence and severity. Acta Odontol Scand 1963;21:533-51.

9. Ainamo J, Bay I. Problems and proposals for recording gingivitis and plaque. Int Dent J. 1975;25(4):229-235.

10. Armstrong R. A. When to use the Bonferroni correction, Ophthal Physiol. Opt. 2014;34(5): 502-508.

11. Krishnan V, Ambili R, Davidovitch Z, Murphy NC. Gingiva and orthodontic treatment. Semin Orthod 2007;13:257-271.

12. Alstad S, Zachrisson BU. Longitudinal study of periodontal condition associated with orthodontic treatment in adolescents. Am J Orthod 1979; 76:277-286.

13. Kilicoglu H, Yildirim M, Polater H. Comparison of the effectiveness of two types of toothbrushes on oral hygiene of patients undergoing orthodontic treatment with fixed appliances. Am J Orthod Dentofacial Orthop 1997; 111:591-594.

14. Cantekin K, Celikoglu M, Karadas M, Yildirim H, Erdem A. Effects of orthodontic treatment with fixed appliances on oral health status: A comprehensive study. Journal of Dental Sciences 2011; 6(4):235-238.

15. Ristic M, VlahovicSvabic M, Sasic M, Zelic O. Clinical and microbiological effects of fixed orthodontic appliances on periodontal tissues in adolescents. Orthod Craniofac Res 2007; 10:187-195.

16. Wolff D, Joers D, Rau P, Dörfer CE. In vitro cleaning efficacy and resistance to insertion test of interdental brushes, Clinical Oral Investigations 2006;10:297-304

17. Poklepovic T, Worthington HV, Johnson TM, Sambungak D, Imai P, Clarkson JE, et al. Interdental brushing for the prevention and control of periodontal diseases and dental caries in adults. Cochrane Database Syst Rev. 2013;12:CD009857

18. Jared H, Zhong Y, Rowe M, Ebisutani K, Tanaka T, Takase N. Clinical trial of a novel interdental brush cleaning system. J Clin Dent. 2005;16(2):47-52. 